05,09,01

\title{
О генерации второй гармоники в неоднородно намагниченных средах
}

\author{
(C) E.A. Караштин ${ }^{1,2}$ \\ ${ }^{1}$ Институт ффизики микроструктур РАН, \\ Нижний Новгород, Россия \\ ${ }^{2}$ Нижегородский государственный университет им. Н.И. Лобачевского, \\ Нижний Новгород, Россия \\ E-mail: eugenk@ipmras.ru
}

\begin{abstract}
Теоретически исследованы новые эффекты генерации оптического сигнала на удвоенной частоте в магнитных средах, распределение намагниченности в которых пространственно неоднородно. Используется подход, основанный на гидродинамическом приближении для описания движения электронов проводимости в поле электромагнитной волны, дополненном уравнениями для среднего спина электронов. В рамках данного подхода продемонстрированы механизмы возникновения эффекта, связанного с тороидным моментом, который был экспериментально обнаружен ранее в системе магнитных частиц с вихревым распределением намагниченности, а также в многослойной магнитной системе (которая является коллинеарной неоднородной магнитной системой). Детально изучен новый эффект, связанный с наличием в неоднородной магнитной системе равновесных спиновых токов. Такой эффект был предсказан из соображений симметрии и может возникать лишь в неколлинеарно намагниченных средах. Показано, что данный эффект имеет резонансный характер с резонансом при частоте накачки, совпадающей с плазменной частотой электронов проводимости. Сделанные оценки восприимчивости на удвоенной частоте для параметров никеля и характерного масштаба изменения намагниченности, равного $10 \mathrm{~nm}$, показывают, что величина восприимчивости вблизи частоты плазменного резонанса составляет при выбранных параметрах порядка $10^{-9} \mathrm{esu}$, что позволяет надеяться на экспериментальное обнаружение эффекта.
\end{abstract}

Работа выполнена при поддержке РФФИ, грант № 16-32-00527.

DOI: 10.21883/FTT.2017.11.45056.15k

\section{1. Введение}

Хорошо известно, что при взаимодействии электромагнитной волны со средой генерация сигнала на удвоенной частоте возможна только в случае, если среда является нецентросимметричной $[1,2]$. При этом центр инверсии может отсутствовать в кристаллической структуре объемного образца (KDP, $\mathrm{LiNbO}_{3}$ и др. $[2,3])$. Такие кристаллы находят применение в качестве нелинейных элементов в оптических схемах и используются как для удвоения частоты излучения, так и для выпрямления (например, получение терагерцового импульса с использованием фемтосекундного лазера [4-6]). С другой стороны, нецентросимметричность может достигаться за счет формы образца. Так, хорошо известно, что сигнал на удвоенной частоте появляется при отражении света от границы полупространства, заполненного центросимметричным материалом [7,8]. Нарушение центра инверсии при этом может характеризоваться нормалью к этой границе. Наличие в материале пространственно-однородного спонтанного магнитного момента приводит к появлению новых слагаемых в тензоре восприимчивости $[9,10]$. Однако, эти слагаемые по-прежнему свзяаны с нормалью к границе. Если рассмотреть неограниченную однородно намагниченную центросимметричную среду, в ней эффекта генерации второй гармоники не будет. ${ }^{1}$

Другой возможностью реализации отсутствия центра инверсии в среде является центросимметричная магнитная среда, заполняющая все пространство, в которой, однако, намагниченность зависит от координаты и при этом ее пространственное распределение не обладает центром инверсии. В наиболее простом случае векторы намагниченности во всех точках при этом коллинеарны. Возникновение нелинейно-оптического эффекта в такой ситуации приписывается тороидному моменту системы $\mathbf{T}=\int[\mathbf{r} \mathbf{M}] d \mathbf{r}$ (который, вообще говоря, хорошо определен только в случае, когда намагниченность М, усредненная по пространственным координатам, равна нулю); данный эффект был обнаружен в плоско-слоистой структуре, состоящей из двух ферромагнитных слоев, разделенных немагнитной прослойкой [11]. Для состояния, когда намагниченности слоев параллельны, тороидный момент равен нулю и генерация второй гармоники возможна лишь за счет эффекта на границе отдельных слоев (этот эффект похож на эффект, связанный с тороидным моментом, поскольку нормаль на границе однородно намагниченного материала характеризует неод-

1 „Слабый“ эффект может возникать из-за того, что распространение электромагнитной волны само по себе нарушает симметрию по отношению к операции инверсии. Очевидно, этот эффект связан с пространственной дисперсией. См., например, [1]. 
нородность распределения М на этой границе; однако, эффект, связанный с тороидным моментом, существует и максимален в случае, когда средняя намагниченность равна нулю, и, кроме того, может возникать в неограниченном ферромагнетике). В антиферромагнитном же состоянии магнитная структура системы не имеет центра инверсии и появляется эффект, связанный с тороидным моментом. Аналогичный эффект был зафиксирован в более сложной системе, представляющей собой двумерную решетку ферромагнитных частиц, в которой удалось создать вихревое распределение намагниченности частиц с одинаковым направлением закрученности вихря во всем массиве [12]. Такой линейный по намагниченности эффект может возникать как за счет простой интерференции сигналов от отдельных границ слоев, так и вследствие особенностей движения электронов в неоднородно намагниченной среде (для плоско-слоистой структуры - в случае, если расстояние между ферромагнитными слоями достаточно мало). Механизмы возникновения эффекта в соответствии со вторым из указанных вариантов, который, как нетрудно понять, является единственным при распространении света в бесконечной непрерывной неоднородно намагниченной среде и поэтому представляет особый интерес, рассмотрены в настоящей работе.

Еще один нелинейно-оптический эффект был предсказан с симметрийной точки зрения в средах, в которых протекают спиновые токи $[13,14]$. Они могут быть индуцированы внешним воздействием (например, в полупроводниках [15]) либо присутствовать в состоянии равновесия; в магнитных наноструктурах спиновый ток в состоянии равновесия возможен лишь при неколлинеарном распределении намагниченности [16-21]. Очевидно, что в обменном приближении спиновый ток может быть записан в низшем порядке по намагниченности $\mathbf{M}$ и ее производным в виде $\sigma_{i k} \sim\left[\mathbf{M}, \frac{\partial \mathbf{M}}{\partial x_{k}}\right]_{i}$ и является квадратичным по М. Тензор спиновых токов $\sigma_{i k}$ меняет знак при операции пространственной инверсии, что делает его удобным для характеризации нецентросимметричности неколлинеарно намагниченных сред. Поляризацию среды на удвоенной частоте, связанную со спиновыми токами, можно записать в виде $P_{l}^{2 \omega}=\beta_{l i k n m} \sigma_{i k} E_{n} E_{m}(\mathbf{E}-$ вектор электрического поля электромагнитной волны). Таким образом, ясно, что наличие спиновых токов в среде приводит к появлению нового вклада в генерацию второй гармоники, пропорционального квадрату намагниченности. Поскольку выражение для поляризации на удвоенной частоте неинвариантно по отношению к вращению М как целого, такой эффект не может быть получен в чисто обменном приближении; для его возникновения необходимо спин-орбитальное взаимодействие. Экспериментально этот эффект в неколлинеарных ферромагнетиках на данный момент не обнаружен. Основные механизмы его возникновения указаны в [22]. В настоящей работе приводится анализ механизмов возникновения указанного эффекта и зависимости его от некоторых параметров среды и частоты распространяющегося в среде излучения.

Заметим здесь, что если проводить дальнейшее разложение по вектору намагниченности среды, можно получить поляризацию на удвоенной частоте в третьем порядке по М вида

$$
P_{l}^{2 \omega}=v_{\text {liknmp }}\left(\mathbf{M}\left[\frac{\partial \mathbf{M}}{\partial x_{i}}, \frac{\partial^{2} \mathbf{M}}{\partial x_{k} \partial x_{n}}\right]\right) E_{m} E_{p} .
$$

Такая поляризация может быть получена в обменном приближении. Фотогальванический эффект (а также эффект выпрямления), аналогичный генерации второй гармоники (второго порядка по электрическому полю волны либо высокочастотному току), был ранее рассчитан для магнитного геликоида [23,24]. Однако, можно ожидать, что величина эффекта третьего порядка по намагниченности и содержащего три ее производных, будет мала. Поэтому в настоящей работе мы ограничиваемся вторым порядком.

\section{2. Теоретическая модель и основные приближения}

В рамках настоящей работы считается, что в оптическом диапазоне частот ответственными за отклик среды при распространении в ней электромагнитной волны являются электроны проводимости. Движение электронов проводимости описывается в рамках гидродинамического приближения, в котором уравнения для скорости $\mathbf{v}$ и концентрации $n$ электронов дополнены законом сохранения их среднего спина $\mathbf{s}$. Система уравнений имеет следующий вид

$$
\begin{gathered}
\mathbf{q}=n \mathbf{v}+\alpha_{S H} e_{i j k} \sigma_{i j} \mathbf{e}_{k}, \\
\sigma_{i j}=s_{i} v_{j}+A\left[\mathbf{M}, \frac{\partial \mathbf{M}}{\partial x_{j}}\right]_{i}, \\
\frac{\partial n}{\partial t}+\operatorname{div} \mathbf{q}=0, \\
\frac{\partial \mathbf{s}}{\partial t}+\operatorname{div}_{\mathbf{r}} \hat{\sigma}+\frac{[\mathbf{s}, \mathbf{M}(\mathbf{r})]}{\tau_{e x}}+\frac{\mathbf{s}-\mathbf{s}_{0}}{\tau_{s}}=0, \\
\frac{\partial \mathbf{q}}{\partial t}+\frac{1}{n}(\mathbf{q}, \nabla) \mathbf{q}=-\frac{e n}{m} \mathbf{E}-\frac{e n}{m} \mathbf{E}^{\prime} \\
+\eta_{H} n[\mathbf{v}, \mathbf{M}]+\eta_{H}^{\prime} n[\mathbf{v}, \mathbf{s}]-\frac{e}{m c}[\mathbf{q}, \mathbf{B}]-\frac{\mathbf{q}}{\tau_{p}}, \\
\operatorname{div} \mathbf{E}^{\prime}=-4 \pi e\left(n-n_{0}\right) .
\end{gathered}
$$

Здесь $\mathbf{q}-$ средний поток электронов, $\alpha_{S H}$ и $\eta_{H}-$ константы спинового и аномального эффектов Холла, $\eta_{H}^{\prime}$ - константа эффекта Холла на собственном среднем магнитном моменте электронов, Е и В - электрическое и магнитное поля волны, $\mathbf{E}^{\prime}-$ поле, наведенное волной в среде, $\tau_{e x}=\frac{\hbar}{2 J}-$ обменное время $(J-$ обменная константа), $\tau_{p}$ и $\tau_{s}$ - время релаксации импульса и спина, 
$e$ и $m-$ заряд и масса электрона, $e_{i j k}-$ антисимметричный тензор Леви-Чивита, $\mathbf{e}_{k}-k$-й орт декартовой системы координат. Намагниченность М считается нормированной на единицу $(|\mathbf{M}|=1)$. Константа $\eta_{H}^{\prime}$, которая определяет эффект Холла электронов на собственном среднем моменте, не связана со спин-орбитальным взаимодействием и поэтому значительно меньше $\frac{\eta_{H}}{n_{0}}$; тем не менее, для полноты картины механизмов возникновения эффектов генерации второй гармоники такой эффект Холла учитывается. В формуле (6) $n_{0}-$ равновесная концентрация электронов проводимости; $\mathbf{s}_{0}$ в формуле (4) - равновесная плотность спина, $\mathbf{s}_{0}=b \mathbf{M}$, где $b=n_{0} \frac{J}{\varepsilon_{F}}\left(\varepsilon_{F}-\right.$ энергия Ферми $)$. Отметим, что для связи спиновой и пространственной степеней свободы электронов в формулу для тока (1) феноменологически вводится обратный спиновый эффект Холла. Аналогичное слагаемое в формуле для спинового тока (2) опущено, поскольку оно дает поправку второго порядка по константе спинового эффекта Холла. Кроме того, в формулу (2) феноменологически введен равновесный спиновый ток с константой $A$, который нельзя получить из данной теории.

Мы решаем систему уравнений (1)-(6) в рамках линейного по константам аномального и спинового эффектов Холла (которые имеют спин-орбитальную природу) приближения. Кроме того, считая намагниченность меняющейся медленно по сравнению с межатомным расстоянием в среде, мы пренебрегаем всеми старшими производными М, оставляя только члены, содержащие первую производную (в первой степени). С другой стороны, мы считаем, что намагниченность меняется быстро по сравнению с длиной электромагнитной волны и пренебрегаем пространственной дисперсией (однако, в уравнении (5) учитывается сила Лоренца в магнитном поле волны; она ответственна за некоторые механизмы генерации второй гармоники, вкладом которых мы затем также пренебрегаем; обсуждение роли таких механизмов приводится ниже). Все величины, входящие в (1)-(6), ищутся в виде разложения по частоте

$$
\begin{gathered}
n=n_{0}+n^{\omega}+n^{2 \omega}+\ldots, \\
\mathbf{v}=\mathbf{v}_{0}+\mathbf{v}^{\omega}+\mathbf{v}^{2 \omega}+\ldots, \\
\mathbf{s}=\mathbf{s}_{0}+\mathbf{s}^{\omega}+\mathbf{s}^{2 \omega}+\ldots, \\
\mathbf{q}=\mathbf{q}_{0}+\mathbf{q}^{\omega}+\mathbf{q}^{2 \omega}+\ldots, \\
\hat{\sigma}=\hat{\sigma}_{0}+\hat{\sigma}^{\omega}+\hat{\sigma}^{2 \omega}+\ldots
\end{gathered}
$$

При этом предполагается, что члены (7)-(11), осциллирующие на частоте $\omega$, пропорциональны первой степени электрического или магнитного поля волны, а члены, осциллирующие на удвоенной частоте, пропорциональны их второй степени. Таким образом, мы пренебрегаем здесь эффектами типа выпрямления, поскольку они лежат за пределами настоящей работы.
Заметим, что $n_{0}-$ константа, являющаяся параметром среды, $\mathbf{s}_{0}=b \mathbf{M}$, как было указано выше, $\left(\sigma_{0}\right)_{i j}=A\left[\mathbf{M}, \frac{\partial \mathbf{M}}{\partial x_{j}}\right]_{i}$ вводится для электронов проводимости феноменологически. Нулевые (равновесные) скорость и поток, вообще говоря, имеют в данном описании разный смысл, поскольку под скоростью подразумевается нормальная скорость электронов, а поток включает в себя также и спин-орбитальные поправки вроде аномальной скорости. Они зависят от граничных условий. В случае бесконечного ферромагнетика нормальная скорость отсутствует: $\mathbf{v}_{0}=0$. Это соответствует наличию равновесного потока электронов, связанного с равновесным спиновым током

$$
\mathbf{q}_{0}=\alpha_{S H} A e_{i j k}\left[\mathbf{M}, \frac{\partial \mathbf{M}}{\partial x_{j}}\right]_{i} \mathbf{e}_{k},
$$

который возникает вследствие, например, спин-орбитального рассеяния на примесях. С таким рассеянием можно связать эффективную силу, действующую на электроны, и ввести эффективное электрическое поле

$$
\mathbf{E}_{0}^{\mathrm{eff}}=-\frac{m \alpha_{S H} A}{e n_{0} \tau_{p}} e_{i j k}\left[\mathbf{M}, \frac{\partial \mathbf{M}}{\partial x_{i}}\right]_{i} \mathbf{e}_{k},
$$

которое будет входить в (5). При этом реального электрического поля в данном случае не возникает. Важно понимать, что потери энергии здесь отсутствуют и система является равновесной. Действительно, при протекании такого равновесного тока энергия вследствие упругого рассеяния электронов на примесях переходит из упорядоченного движения в хаотическое, т.е. электронный газ нагревается. Однако, в то же время при образовании потока электронов (12) энергия за счет силы, описываемой эффективным полем (13), переходит из хаотического движения электронов в упорядоченное, что в равновесии в точности компенсирует указанный выше обратный переход энергии. Таким образом, два диссипативных по своей природе процесса приводят к существованию в равновесии потока электронов без нарушения закона сохранения энергии.

В случае ограниченного ферромагнетика в процессе установления равновесия поток электронов приведет к разделению зарядов. Эти заряды создадут электрическое поле, которое компенсирует эффективное поле (13). Среда при этом будет электрически поляризована, в соответствии с предсказаниями [25]. За счет действия на электроны возникшего поля они приобретут нормальную равновесную скорость

$$
\mathbf{v}_{0}=-\frac{\alpha_{S H}}{n_{0}} e_{i j k}\left[\mathbf{M}, \frac{\partial \mathbf{M}}{\partial x_{j}}\right]_{i} \mathbf{e}_{k},
$$

которая компенсирует поправки типа аномальной скорости, и средний поток электронов $\mathbf{q}_{0}=0$.

Поляризация среды на удвоенной частоте $\mathbf{P}^{2 \omega}$ легко может быть найдена по формуле

$$
\mathbf{P}^{2 \omega}=-\frac{e}{2 i \omega} \mathbf{q}^{2 \omega}
$$


Таким образом, для нахождения отклика среды на удвоенной частоте необходимо вычислить поток электронов на этой частоте $\mathbf{q}^{2 \omega}$.

\section{3. Генерация второй гармоники неколлинеарно намагниченной системой}

3.1. Т ороидный момент. Для нахождения вкладов в генерацию сигнала на удвоенной частоте, связанных с тороидным моментом системы, мы вычисляем в рамках описанных выше приближений поток электронов, линейный по намагниченности. Он определяется выражением

$$
\mathbf{q}_{1}^{2 \omega}=u_{1}(\mathbf{E}, \operatorname{rot} \mathbf{M}) \mathbf{E}+u_{2}[\mathbf{E},(\mathbf{E}, \nabla) \mathbf{M}] .
$$

Коэффициенты $u_{1}$ и $u_{2}$ определяются следующими выражениями:

$$
\begin{aligned}
u_{1}=\frac{\left(\frac{e}{m}\right)^{2}}{\omega\left(\omega-\frac{\omega_{p}^{2}}{\omega}-\frac{i}{\tau_{p}}\right)^{3}} \\
\times\left(\left(b \alpha_{S H}\left(i \omega+\frac{1}{\tau_{p}}\right)\right)-\left(n_{0}\left(\eta_{H}+b \eta_{H}^{\prime}\right)\right)\right), \\
u_{2}=\frac{\omega\left(\omega-\frac{\omega_{p}^{2}}{\omega}-\frac{i}{\tau_{p}}\right)^{2}}{\left(1+\tau_{e x}^{2}\left(i \omega+\frac{1}{\tau_{s}}\right)^{2}\right)} \\
\times\left(\alpha_{S H}+\frac{i \tau_{e x}^{2}\left(i \omega+\frac{1}{\tau_{s}}\right)}{\left.2 \omega-\frac{\omega_{p}^{2}}{2 \omega}-\frac{i}{\tau_{p}}\right)}\right. \\
\left.+\frac{\left(n_{0}\left(\eta_{H}+b \eta_{H}^{\prime}\right)-i b \alpha_{S H}\left(\omega+\frac{\omega_{p}^{2}}{\omega}-\frac{i}{\tau_{p}}\right)\right.}{\left(\omega-\frac{\omega_{p}^{2}}{\omega}-\frac{i}{\tau_{p}}\right)\left(2 \omega-\frac{\omega_{p}^{2}}{2 \omega}-\frac{i}{\tau_{p}}\right)}\right) .
\end{aligned}
$$

Эффекты, определяемые формулой (15), соответствуют эффектам, которые в $[11,12]$ описываются с помощью тороидного момента. Действительно, в магнитном вихре и тороидный момент, и, например, $\operatorname{rot} \mathbf{M}$ направлены перпендикулярно вихрю и имеют одинаковую симметрию по отношению к операциям пространственной инверсии и обращения времени. Однако, при описании движения электронов (в отличие от средних параметров среды) удобнее пользоваться дифференциальными характеристиками. Заметим, что несмотря на то что, в соответствии с теоремой Стокca, среднее значение $\operatorname{rot} \mathbf{M}$ за счет границ обращается в вихре в ноль, эффект типа (15) не должен пропадать, поскольку электроны проводимости движутся внутри объема материала и граница может на них

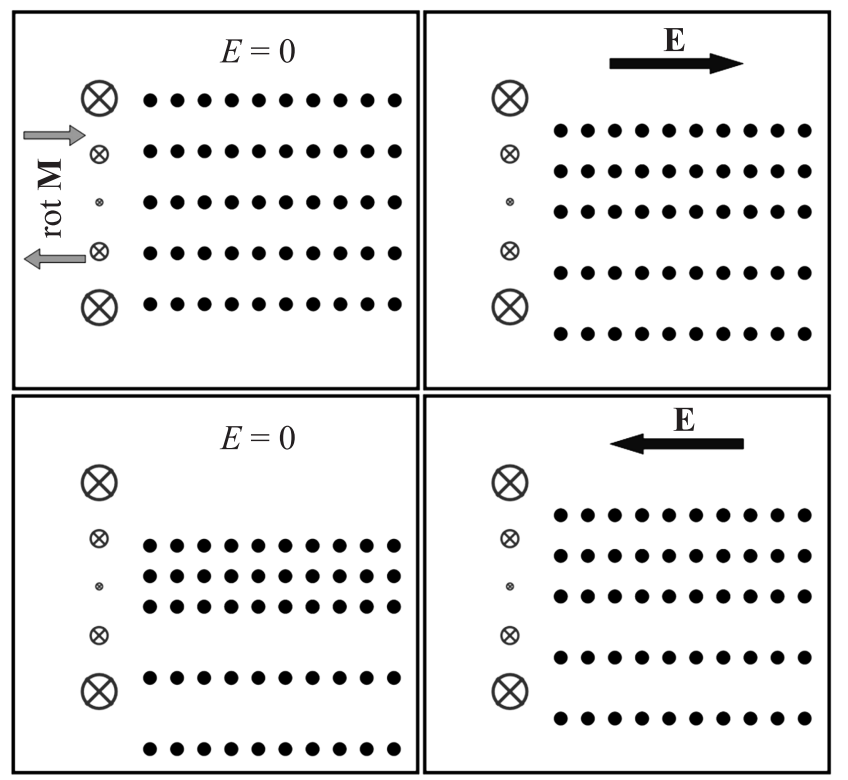

Рис. 1. Поперечные осцилляции электронов проводимости в среде с неоднородной намагниченностью. Показаны положения электронов в моменты времени, соответствующие разным фазам поля.

влиять лишь за счет отражения, т.е. существенно иным способом.

Механизмов возникновения линейных по намагниченности эффектов довольно много. Во-первых, аномальный эффект Холла, возникающий при осцилляции электронов в электрическом поле волны, приводит к смещению их в поперечном направлении. В неоднородно намагниченной среде величина этого смещения зависит от локальной намагниченности, что приводит к появлению пространственно-неоднородной концентрации электронов, осциллирующей на частоте волны. Совместно с колебаниями электронов в поле волны это дает ток на удвоенной частоте, который определяется коэффициентом $u_{1}$ (второе слагаемое в правой части (16)). Данный механизм как один из самых простых проиллюстрирован на рис. 1. Из рисунка видно, что в результате аномального эффекта Холла в неоднородно намагниченной среде возникает осциллирующая плотность электронов. Второй механизм также связан с появлением неоднородной концентрации электронов, но возникает в результате переноса спина осциллирующими электронами. Такой поток спина конвертируется в неоднородный электрический ток (на частоте волны) за счет спинового эффекта Холла. Неоднородность этого тока, возникающая из неоднородности спиновой поляризации, и приводит к возникновению неоднородности концентрации электронов, колеблющейся на частоте волны. Это дает вклад в $u_{1}$, определяющийся первым слагаемым в правой части (16). В-третьих, „адиабатический“ перенос спина движущимися в поле волны спин-поляризованными электронами приводит к возникновению потока спина 
на удвоенной частоте, который затем конвертируется в электрический ток посредством спинового эффекта Холла. Это дает вклад в $u_{2}$, который определяется первым слагаемым, пропорциональным $\alpha_{S H}$, в правой части (17). Еще один механизм связан с возникновением пространственно-неоднородной скорости электронов на частоте $\omega$ из-за аномального эффекта Холла, которая, в свою очередь, приводит к ненулевой конвективной производной от скорости и, как следствие, к появлению скорости на удвоенной частоте. Этот механизм дает вклад в $u_{2}$ в виде слагаемого, содержащего $n_{0}\left(\eta_{H}+b \eta_{H}^{\prime}\right)$, в правой части (17). Экранировка, описываемая уравнением (6), приводит к возникновению резонансов на частоте $\omega_{p}$ и $\frac{\omega_{p}}{2}$ и, кроме того, является причиной возникновения второго слагаемого, содержащего $\alpha_{S H}$, в правой части (17). Эффект Холла на собственном магнитном моменте электронов дает, во-первых, поправку к константе аномального эффекта Холла и, во-вторых, являясь сам по себе механизмом возникновения нелинейности в уравнении (5), приводит к возникновению дополнительных скорости и потока электронов на удвоенной частоте, которые дают слагаемое, содержащее $n_{0} \eta_{H}^{\prime}$, в правой части (17).

Для оценки величины эффекта, определяемого формулой (15), и сравнения разных механизмов его возникновения, будем использовать следующие материальные параметры (по умолчанию, если не указано иное). В качестве ферромагнитного материала выбран никель. Обменная константа составляет $J=0.25 \mathrm{eV}[26]$, откуда следует $\tau_{e x}=1.3 \cdot 10^{-15} \mathrm{~s}$. При этом времена релаксации импульса и спина выбираются равными $\tau_{p}=10^{-14} \mathrm{~s}, \tau_{s}=10^{-13} \mathrm{~s}$. Энергия Ферми $\varepsilon_{F}=4.3 \mathrm{eV}$, равновесная плотность электронов $n_{0} \approx 4.1 \cdot 10^{22} \mathrm{~cm}^{-3}$. Намагниченность насыщения никеля $M_{s}=520 \mathrm{Gs}$ [28]. Угол аномального эффекта Холла составляет по порядку величины $\alpha_{H} \sim 10^{-2}[26,28]$, что позволяет оценить величину константы $\eta_{H}=\frac{\alpha_{H}}{\tau_{p}} \sim 10^{12} \mathrm{~s}^{-1}$. Константа эффекта Холла на собственном магнитном моменте электронов имеет другую размерность, поскольку величина $\mathbf{s}$ введена как плотность (безразмерного) спина. Ее можно оценить из силы Лоренца: $n_{0} \eta_{H}^{\prime} \sim 0.9 \cdot 10^{10} \mathrm{~s}^{-1}$. Данная величина на два порядка меньше константы аномального эффекта Холла. Угол спинового эффекта Холла для $\mathrm{Ni}$, в соответствии с [29], $\alpha_{S H}=0.076$.

На рис. 2, $а$ показана зависимость от частоты модулей $u_{1}$ и $u_{2}$. Во всем диапазоне частот $\left|u_{2}\right|$ больше, чем $\left|u_{1}\right|$. Исключение составляет область резонанса при $\omega_{p}$, где они сравнимы. Для $u_{2}$ хорошо видны три резонансных пика: при $\tau_{e x}^{-1}$, при $\frac{\omega_{p}}{2}$ и при $\omega_{p}$ (в порядке увеличения частоты). Максимальная величина, существенно превосходящая другие, наблюдается при $\omega_{p}$. Рис. $2, b$ содержит зависимость двух вкладов в $u_{1}$ (см. формулу (16)) от частоты. Хорошо видно, что слагаемое, связанное со спиновым эффектом Холла, во всем диапазоне частот заметно превосходит вклад от аномального эффекта Холла. На рис. 2, с показана
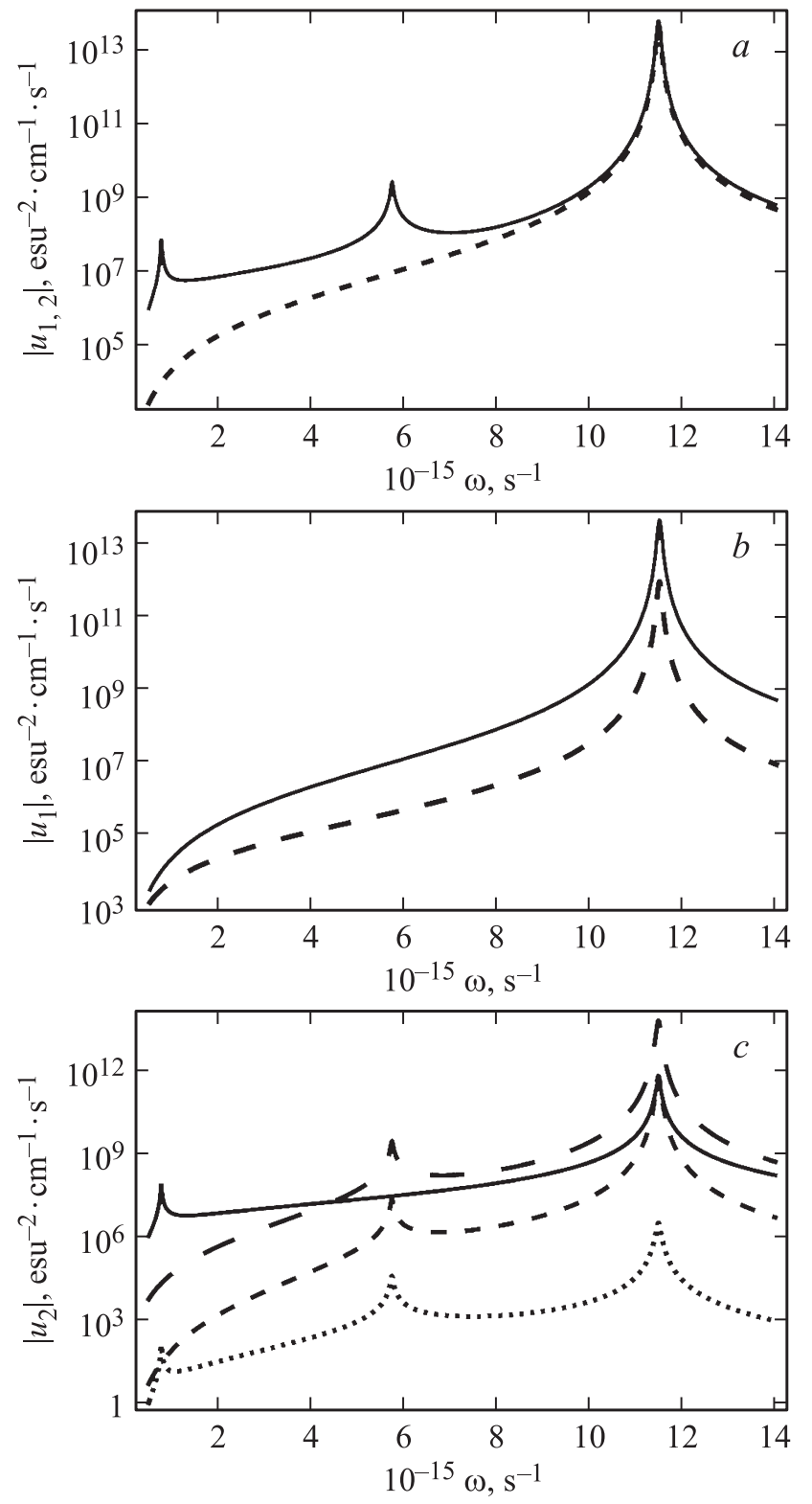

Рис. 2. Зависимость от частоты $(a)$ модулей $u_{1}$ (штриховая линия) и $u_{2}$ (сплошная линия); $(b)$ модулей вкладов в $u_{1}$ (сплошная линия - первое слагаемое в правой части (16), штриховая линия - второе слагаемое в правой части (16)); (c) модулей вкладов в $u_{2}$ (сплошная линия - первое слагаемое с $\alpha_{S H}$ в правой части (17), штриховая линия (длинный штрих) - второе слагаемое с $\alpha_{S H}$ в правой части (17), штриховая линия (короткий штрих) - слагаемое, содержащее $n_{0}\left(\eta_{H}+b \eta_{H}^{\prime}\right)$, в правой части (17), пунктирная линия слагаемое, содержащее $n_{0} \eta_{H}^{\prime}$, в правой части (17)).

зависимость от частоты четырех слагаемых в $u_{2}$ (формула (17)). Видно, что механизм, связанный с эффектом Холла на собственном магнитном моменте электронов, существенно меньше других слагаемых. Вклад, связанный с аномальным эффектом Холла, также мал во всем диапазоне частот по сравнению со слагаемыми, связанными со спиновым эффектом Холла. При низких 
частотах (резонанс при $\tau_{e x}^{-1}$ и в окрестности этой точки) величина $u_{2}$ определяется механизмом, возникающим в результате „адиабатического“ переноса спина (первое слагаемое с $\alpha_{S H}$ в (16)). С увеличением частоты растет вклад от механизма, связанного с экранировкой неоднородной плотности электронов (второе слагаемое с $\alpha_{S H}$ в (16)), и этот механизм является доминирующим в окрестностях $\frac{\omega_{p}}{2}$ и $\omega_{p}$. При частоте, заметно превышающей $\omega_{p}$, максимальный вклад дает опять механизм, связанный с „адиабатическим“ переносом спина (не показано на рис. 2,c). Таким образом, можно сделать вывод, что наиболее существенными при параметрах реальных материалов являются механизмы, связанные со спиновым эффектом Холла.

3.2. Спиновы й ток. Для того, чтобы получить эффект генерации второй гармоники, связанный со спиновым током в системе, найдем поток на удвоенной частоте второго порядка по намагниченности. Вычисления показывают, что он имеет вид

$$
\begin{aligned}
\mathbf{q}_{2}^{2 \omega}= & w_{1}[\mathbf{E},[\mathbf{M},(\mathbf{E}, \nabla) \mathbf{M}]] \\
& +w_{2}\left[\left[\left(e_{i j k}\left[\mathbf{M}, \frac{\partial \mathbf{M}}{\partial x_{j}}\right]_{i} \mathbf{e}_{k}\right), \mathbf{B}\right], \mathbf{B}\right],
\end{aligned}
$$

где $w_{1}$ и $w_{2}$ определяются выражениями

$$
\begin{aligned}
w_{1}= & \frac{-\left(\frac{e}{m}\right)^{2} \tau_{e x} b}{\left(1+\left(i \omega+\frac{1}{\tau_{s}}\right)^{2} \tau_{e x}^{2}\right)\left(\omega-\frac{\omega_{p}^{2}}{\omega}-\frac{i}{\tau_{p}}\right)^{2}} \\
& \times\left(\alpha_{S H}+\frac{i n_{0} \eta_{H}^{\prime}}{2 \omega-\frac{\omega_{p}^{2}}{2 \omega}-\frac{i}{\tau_{p}}}\right), \\
w_{2}= & \frac{-\left(\frac{e}{m}\right)^{2} \alpha_{S H} A}{c^{2}\left(\omega-\frac{\omega_{p}^{2}}{\omega}-\frac{i}{\tau_{p}}\right)\left(2 \omega-\frac{\omega_{p}^{2}}{2 \omega}-\frac{i}{\tau_{p}}\right)} .
\end{aligned}
$$

Выражение (20) получено для случая бесконечного ферромагнетика, в котором имеется $\mathbf{q}_{0}$, заданный формулой (12). Он возникает из силы Лоренца, действующей на поток электронов в магнитном поле волны (во втором порядке). Поскольку кроме малой константы, связанной со спин-орбитальным взаимодействием, данный вклад содержит малый параметр $\left(\frac{v}{c}\right)^{2}(v-$ характерная скорость электронов), он должен быть мал по сравнению с первым слагаемым в (18), которое определяется коэффициентом (19). Это слагаемое возникает в результате появления у электронов „неадиабатического“ спина на частоте $\omega$ при движении в поле волны, связанного с тем, что в неколлинеарной среде спин при смещении электронов прецессирует вокруг локального магнитного момента. Совместно со скоростью на частоте волны этот спин приводит к возникновению спинового тока на удвоенной частоте, который затем конвертируется в

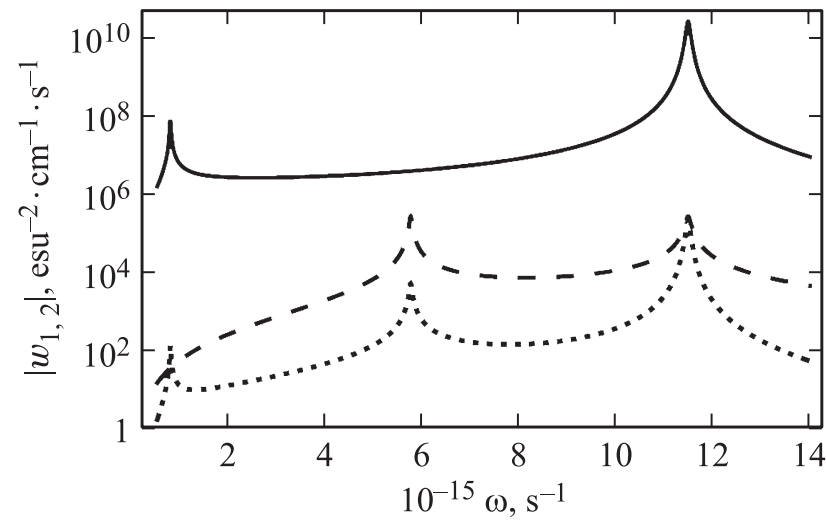

Рис. 3. Зависимость модуля $w_{1}$, первое (сплошная линия) и второе (пунктирная линия) слагаемые в (19), а также модуля $w_{2}$ (штриховая линия) от частоты для параметров никеля.

электрический с помощью обратного спинового эффекта Холла. Данный механизм описан в [22]. Заметим, что аномальный эффект Холла не дает вклада в эффекты второго порядка по намагниченности, в отличие от эффекта Холла на собственном магнитном моменте электронов. Это связано с тем, что мы ограничиваемся линейными по $\eta_{H}$ и $\alpha_{S H}$ слагаемыми. В то же время, эффект Холла на спине электронов дает дополнительную нелинейность. В результате спин электронов и их скорость, осциллирующие на частоте $\omega$, порождают скорость на частоте $2 \omega$, что приводит к возникновению тока на этой частоте.

Для оценки и количественного сравнения разных вкладов в (18) воспользуемся, как и ранее, параметрами никеля. Константу $A$, определяющую величину равновесного спинового тока, можно оценить так, как это сделано для константы флексо-магнитоэлектрического эффекта в работе [31]: $A \approx 2 \cdot 10^{22} \mathrm{~s}^{-1} \mathrm{~cm}^{-1}$.

На рис. 3 показаны зависимости модулей первого и второго слагаемых в (19), а также модуля выражения (20) от частоты волны. Видно, что оба слагаемых в (19) имеют относительно небольшой по абсолютной величине резонанс на частоте $\tau_{e x}^{-1}$ и значительный резонанс на $\omega_{p}$. Кроме того, второе слагаемое также имеет резонанс на $\frac{\omega_{p}}{2}$. Величина $w_{2}$ имеет резонансы лишь на $\omega_{p}$ и $\frac{\omega_{p}}{2}$. Сравнение кривых на рис. 3 показывает, что действительно вклад второго слагаемого в $w_{1}$, связанного с эффектом Холла на собственном магнитном моменте электронов, существенно (примерно на пять порядков) меньше, чем вклад первого слагаемого в (19). Поэтому этот эффект можно не учитывать. Кроме того, видно, что величина $w_{2}$ также существенно меньше, чем $w_{1}$. Это подтверждает рассуждения о малости данного вклада, который также можно не учитывать. Нетрудно видеть, что увеличение времени релаксации импульса $\tau_{p}$ приводит к квадратичному росту обоих слагаемых в (19) в резонансе, в то время, как ве- 

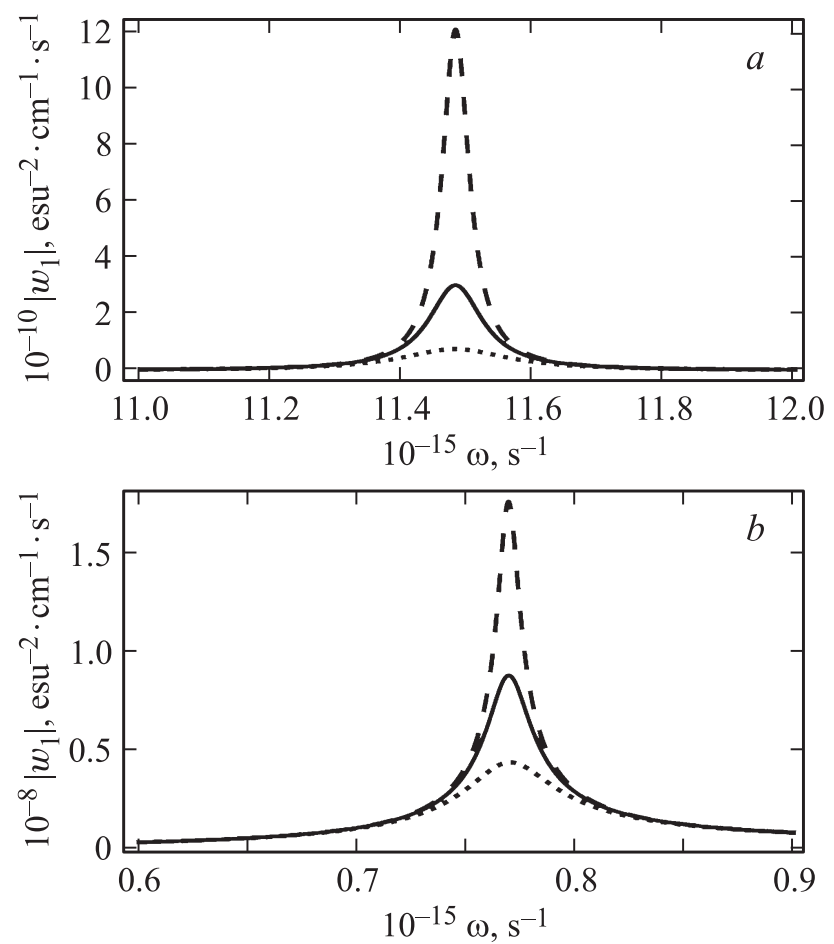

Рис. 4. Зависимость модуля $w_{1}$ от частоты $(a)$ в окрестности плазменного резонанса для $\tau_{p}=10^{-14} \mathrm{~s}$ (сплошная линия), $\tau_{p}=2 \cdot 10^{-14} \mathrm{~s}$ (штриховая линия), $\tau_{p}=0.5 \cdot 10^{-14} \mathrm{~s}$ (пунктирная линия); $(b)$ в окрестности обменного резонанса $\tau_{s}=10^{-13} \mathrm{~s}$ (сплошная линия), $\tau_{s}=2 \cdot 10^{-13} \mathrm{~s}$ (штриховая линия), $\tau_{s}=0.5 \cdot 10^{-13} \mathrm{~s}$ (пунктирная линия). Другие параметры соответствуют никелю.

личина (20) растет линейно. Обменный резонанс на частоте $\tau_{e x}^{-1}$ растет с увеличением времени релаксации спина $\tau_{s}$ одинаково для обоих слагаемых в (19). Таким образом, можно сделать вывод о том, что для реальных параметров достаточно ограничиться первым слагаемым в $w_{1}$ (формула (19)), определяемым спиновым эффектом Холла, и пренебречь всеми другими механизмами, дающими вклад в (18).

На рис. 4 представлена зависимость модуля $w_{1}$ от частоты для разных значений времени релаксации. Как и следовало ожидать, с увеличением $\tau_{p}$ величина эффекта на частоте $\omega_{p}$ растет (рис. $\left.4, a\right)$. При этом величина $\tau_{p}$ практически не влияет на резонанс на частоте $\tau_{e x}^{-1}$. С другой стороны, величина $\tau_{s}$ не влияет на резонанс на плазменной частоте; при этом увеличение $\tau_{s}$ приводит к росту $\left|w_{1}\right|$ в области резонанса при $\tau_{e x}^{-1}$ (рис. $4, b)$. На рис. 5 показана величина молуля $w_{1}$ для разных материалов (никель, пермаллой, кобальт, железо). Для каждого из материалов наблюдается два резонанса: небольшой по амплитуде при $\tau_{e x}^{-1}$ и значительный при $\omega_{p}$. Величина резонанса при $\omega_{p}$ близка по величине для всех материалов: отношение максимальной величины $\left(\left|w_{1}\right| \approx 2.2 \cdot 10^{11} \mathrm{esu}^{-1} \mathrm{~cm}^{-1} \mathrm{~s}^{-1}\right)$ в кобальте к минимальной в никеле составляет порядка 10 . Величина эффекта в кобальте максимальна, поскольку величина обменной константы $J$ в кобальте заметно больше, чем в никеле. При этом угол спинового эффекта Холла в кобальте, хоть и меньше, чем в никеле, но больше, чем в других материалах.

Оценим величину нелинейной восприимчивости для никеля (отношения модуля поляризации на удвоенной частоте к квадрату поля волны на основной частоте) в резонансе $\left(\omega=\omega_{p}\right)$. Для этого возьмем характерный масштаб неоднородности намагниченности $L_{M} \approx 10 \mathrm{~nm}$ (такую неоднородность представляется реальным создать наноструктурированием материала). Подставляя параметры никеля и выбранный масштаб в формулы (18)-(20) и используя формулу (14), получим $\frac{\left|\mathbf{P}^{2 \omega}\right|}{|\mathbf{E}|^{2}} \approx 0.6 \cdot 10^{-9}$ esu. Это значение по порядку величины сравнимо с восприимчивостью такого материала, как дигидроортофосфат калия (KDP) [2,3]. Поэтому экспериментальное наблюдение данного эффекта представляется реальным.

Для эксперимента по обнаружению эффекта, связанного со спиновыми токами, в генерации второй гармоники удобно использовать многослойную искусственную ферромагнитную структуру. В такой структуре можно реализовать неколлинеарное распределение намагниченности, которое будет обладать значительной величиной неоднородности и контролироваться приложением внешнего магнитного поля [31]. В системе, состоящей из двух магнитных слоев, спиновый ток приобретает вид $\sigma_{i j} \sim\left[\mathbf{M}_{1}, \mathbf{M}_{2}\right]_{i} n_{j}$, где $\mathbf{n}-$ вектор нормали к слоям. $\mathrm{B}$ применении к поляризации на удвоенной частоте $\mathbf{P}^{2 \omega}$ это означает, что в системе, кроме векторов $\mathbf{M}_{1}$ и $\mathbf{M}_{2}$, появляется дополнительный вектор $\left[\mathbf{M}_{1}, \mathbf{M}_{2}\right]$, который входит в выражение для поляризации так же, как и намагниченности. Используя таблицу генерируемого намагниченной системой сигнала на удвоенной частоте в зависимости от поляризации падающего излучения и направления намагниченности [32], нетрудно видеть, что в случае, когда оба вектора $\mathbf{M}_{1}$ и $\mathbf{M}_{2}$ лежат в плоско-

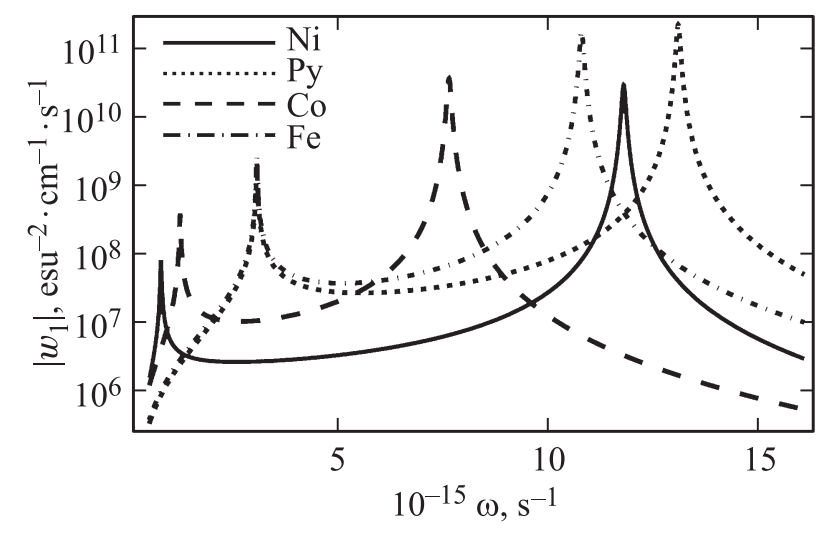

Рис. 5. Зависимость модуля $w_{1}$ от частоты для разных материалов (сплошная линия - никель). Параметры взяты из $[27,28]$. 


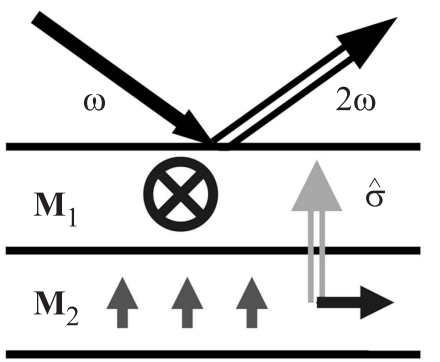

Рис. 6. Схема возможного эксперимента по измерению генерации второй гармоники в системе с равновесным спиновым током.

сти падения линейно поляризованной волны (рис. 6), $p$-поляризованного сигнала на удвоенной частоте нет. $\mathrm{B}$ то же время при появлении вектора $\left[\mathbf{M}_{1}, \mathbf{M}_{2}\right]$ указанный сигнал должен появиться. Таким образом, комбинации $p-p$ и $s-p$ падающего излучения и генерируемой на удвоенной частоте волны являются оптимальными для наблюдения нового эффекта, связанного со спиновым током в неколлинеарно намагниченной системе.

\section{4. Заключение}

В настоящей работе в гидродинамическом приближении изучены механизмы возникновения линейных и квадратичных по намагниченности эффектов в генерации второй гармоники в средах, в которых отсутствие центра инверсии достигается за счет пространственной неоднородности распределения намагниченности. Дано объяснение ранее наблюдавшемуся эффекту, связанному с наличием среднего тороидного момента системы. Помимо интерференционных эффектов, которые могут играть роль лишь в случае, если центросимметричность нарушается в некоторых областях пространства за счет других материальных параметров (не намагниченности, a, например, плотности вещества), возникают эффекты, связанные с особенностями движения электронов проводимости в неоднородно намагниченной среде. К таким особенностям можно отнести осцилляции плотности электронов на частоте распространяющейся в среде волны, осцилляции среднего спина электронов из-за переноса спина под действием поля волны, возникновение скорости электронов на удвоенной частоте вследствие аномального эффекта Холла, зависящего от пространственных координат, а также эффект Холла на магнитном моменте, создаваемом самими электронами.

Исследован новый, ранее не наблюдавшийся эффект второго порядка по намагниченности, связанный с наличием в неколлинеарно намагниченной среде равновесного спинового тока. Данный эффект возникает вследствие „неадиабатической“ поправки к среднему спину электронов, перпендикулярной как намагниченности, так и направлению ее изменения. Эта поправка приводит к возникновению спинового тока на удвоенной частоте, который трансформируется в электрический посредством обратного спинового эффекта Холла. Продемонстрировано, что эффект имеет резонансный характер и значительно усиливается вблизи плазменной частоты электронов проводимости. Выполнены оценки эффекта для различных материалов (никель, пермаллой, кобальт, железо) и даны рекомендации по его экспериментальному наблюдению.

Показано, что для реалистичных значений параметров как линейный, так и квадратичный по намагниченности эффекты, содержащие ее производную, определяются в основном обратным спиновым эффектом Холла.

Автор выражает благодарность А.А. Фраерману, Т.В. Мурзиной и А.Н. Караштину за полезные обсуждения.

\section{Список литературы}

[1] Л.Д. Ландау, Е.М. Лифшиц. Электродинамика сплошных сред. Наука, М. (1982).

[2] R.W. Boyd. Nonlinear Optics. Academ. Press, Rochester, N. Y. (2008).

[3] Г.Г. Гурзадян, В.Г. Дмитриев, Д.Н. Никогосян. Нелинейнооптические кристаллы. Свойства и применение в квантовой электронике. Радио и связь, М. (1991).

[4] K. Reimann. Rep. Prog. Phys. 70, 1597 (2007).

[5] S.B. Bodrov, M.I. Bakunov, M. Hangyo. J. Appl. Phys. 104, 093105 (2008).

[6] S.B. Bodrov, A.N. Stepanov, M.I. Bakunov, B.V. Shishkin, I.E. Ilyakov, R.A. Akhmedzhanov. Opt. Express 17, 3, 1871 (2009).

[7] Y.R. Shen. Ann. Rev. Mater. Sci. 16, 69 (1986).

[8] P. Guyot-Sionnest, W. Chen, Y.R. Shen. Phys. Rev. B 33, 8254 (1986).

[9] R.-P. Pan, H.D. Wei, Y.R. Shen. Phys. Rev. B 39, 1229 (1989).

[10] H.A. Wierenga, M.W.J. Prins, D.L. Abraham, Th. Rasing. Phys. Rev. B 50, 1282 (1994).

[11] I.A. Kolmychek, V.L. Krutyanskiy, T.V. Murzina, M.V. Sapozhnikov, E.A. Karashtin, V.V. Rogov, A.A. Fraerman. J. Opt. Soc. Am. B 32, 331 (2015).

[12] V.L. Krutyanskiy, I.A. Kolmychek, B.A. Gribkov, E.A. Karashtin, E.V. Skorohodov, T.V. Murzina. Phys. Rev. B 88, 094424 (2013).

[13] J. Wang, B.-F. Zhu, R.-B. Liu. Phys. Rev. Lett. 104, 256601 (2010).

[14] J. Wang, S.-N. Ji, B.-F. Zhu, R.-B. Liu. Phys. Rev. B 86, 045215 (2012).

[15] L.K. Werake, H. Zhao. Nature Phys. 6, 875 (2010).

[16] E. Sonin. Adv. Phys. 59, 181 (2010).

[17] J. Wang, K.S. Chan. Phys. Rev. B 74, 035342 (2006).

[18] J. Wang, K.S. Chan. J. Phys.: Condens. Matter. 19, 236215 (2007).

[19] Y.G. Shen, Z.H. Yang. Europhys. Lett. 78, 17003 (2007).

[20] W. Chen, P. Horsch, D. Manske. Phys. Rev. B 89, 064427 (2014).

[21] P. Bruno, V.K. Dugaev. Phys. Rev. B 72, 241302(R) (2005).

[22] E.A. Karashtin, A.A. Fraerman. https://arxiv.org/abs/1704.02229 
[23] А.А. Фраерман, О.Г. Удалов. Письма в ЖЭТФ 87, 3, 187 (2008).

[24] A.A. Fraerman, O.G. Udalov. Phys. Rev. B 77, 094401 (2008).

[25] В.Г. Барьяхтар, В.А. Львов, Д.А. Яблонский. Письма в ЖЭТФ 37, 12, 565 (1983).

[26] C.S. Wang, J. Callaway. Phys. Rev. B 9, 4897 (1974).

[27] А.П. Бабичев, Н.А. Бабушкина, А.М. Братковский, М.Е. Бродов, М.В. Быстрое, Б.В. Виноградов, Л.И. Винокурова, Э.Б. Гельман, А.П. Геппе, И.С. Григорьев, К.Г. Гуртовой, В.С. Егоров, А.В. Елецкий, Л.К. Зарембо, В.Ю. Иванов, В.Л. Ивашинцева, В.В. Игнатьев, Р.М. Имамов, А.В. Инюшкин, Н.В. Кадобнова, И.И. Карасик, К.-А. Кикоин, В.А. Криворучко, В.М. Кулаков, С.Д. Лазарев, Т.М. Лифшиц, Ю.Э. Любарский, С.В. Марин, И.А. Маслов, Е.3. Мейлихов, А.И. Мигачев, С.А. Миронов, А.Л. Мусатов, Ю.П. Никитин, Л.А. Новицкий, А.И. Обухов, В.И. Ожогин, Р.В. Писарев, Ю.В. Писаревский, В.С. Птускин, А.А. Радциг, В.П. Рудаков, Б.Д. Сумм, Р.А. Сюняев, М.Н. Хлопкин, И.Н. Хлюстиков, В.М. Черепанов, А.Г. Чертов, В.Г. Шапиро, В.М. Шустряков, С.С. Якимов, В.П. Яновский. Физические величины. Энергоатомиздат, М. (1991).

[28] S. Zhang. Phys. Rev. Lett. 85, 393 (2000).

[29] H. Wang, C. Du, P. Chris Hammel, F. Yang. Appl. Phys. Lett. 104, 202405 (2014).

[30] Е.А. Караштин, А.А. Фраерман. ФТТ 58, 11, 2149 (2016).

[31] E.S. Demidov, N.S. Gusev, L.I. Budarin, E.A. Karashtin, V.L. Mironov, A.A. Fraerman. J. Appl. Phys. 120, 173901 (2016).

[32] A.A. Rzhevsky, B.B. Krichevtsov, D.E. Burgler, C.M. Schneider. Phys. Rev. B 75, 144416 (2007). 